\title{
Semiconductor nanowire building blocks: From flux line pinning to artificial photosynthesis
}

\section{Peidong Yang}

The following article is an edited transcript of the MRS Medal Lecture presented by Peidong Yang on November 30, 2011 at the 2011 Materials Research Society Fall Meeting in Boston. The MRS Medal is awarded for a specific outstanding recent discovery or advancement that has a major impact on the progress of a materials-related field. Yang received the award for "outstanding contributions in the creative synthesis and assembly of semiconductor nanowires and their heterostructures, and innovations in nanowire-based photonics, thermoelectrics, solar energy conversion, and nanofluidic applications."

Semiconductor nanowires, by definition, typically have nanoscale cross-sectional dimensions, with lengths spanning from hundreds of nanometers to millimeters. These subwavelength structures represent a new class of semiconductor materials for investigating light generation, propagation, detection, amplification, and modulation. After more than a decade of research, nanowires can now be synthesized and assembled with specific compositions, heterojunctions, and architectures. This has led to a host of nanowire photonic and electronic devices, including photodetectors, chemical and gas sensors, waveguides, LEDs, microcavity lasers, and nonlinear optical converters. Nanowires also represent an important class of nanostructure building blocks for photovoltaics as well as direct solar-to-fuel conversion because of their high surface area, tunable bandgap, and efficient charge transport and collection. This article gives a brief history of nanowire research for the past two decades and highlights several recent examples in our lab using semiconductor nanowires and their heterostructures for the purpose of solar energy harvesting and waste heat recovery.

\section{Introduction}

My personal journey in nanowire research started with Professor Charles Lieber at Harvard University, where I investigated flux line pinning in high $\mathrm{T}_{\mathrm{c}}$ superconductors using nanowires as pinning centers. More than 15 years later, I devote most of my efforts in studying these nanostructures for energy conversion and storage purposes.

I would like to acknowledge the pioneering contribution of R.S. Wagner at Bell Laboratories. Much of our nanowire research today relies on the very powerful method developed by Wagner, known as the "vapor-liquid-solid process" (VLS). ${ }^{1}$ In 1964, Wagner described the VLS growth of silicon microand nanoscale wires or whiskers and pointed out that one of the catalysts that can be used is gold. Gold is often used today to grow silicon nanowires, as well as copper, nickel, and, more recently, aluminum. Wagner also noted that nanowires can grow into different cross directions. Over the past 10 years, there have been thousands of papers on silicon nanowire growth, growth direction control, and the use of different catalysts and substrates, all based on Wagner's original concept. ${ }^{2}$ Wagner's work in the 1960s set the foundation for much of today's nanowire research.

My first nanowire experiments were in the early 1990s, when many of us were still working on high- $\mathrm{T}_{\mathrm{c}}$ superconductors (HTSC). The subject of my PhD thesis was to find a way to introduce linear defects within high-temperature superconductors, in the hope of increasing the critical current density. Our approach was to introduce single-crystalline nanowires into a high- $\mathrm{T}_{\mathrm{c}}$ cuprate superconductor to make a composite, to create stable linear tracks, and to increase the critical current density by "pinning" the flux lines. ${ }^{3}$ This work paralleled much of the work conducted at the time using fast ion irradiation to create linear defects within high-temperature superconductors. That was the start of my research in this very exciting area, and it 
was also the beginning of nanowire research in Lieber's group. Shortly after, the landmark paper by Morales and Lieber (1998) 4 introduced the laser ablation method for the growth of silicon nanowires, once again based on the VLS process. In this case, the vapor was generated using laser ablation. After that, there was significant research using VLS processes to grow semiconductor nanostructures of many different compositions. Many different vapor deposition methods were employed, including chemical vapor deposition (CVD) and several physical vapor deposition techniques.

After joining the University of California, Berkeley faculty in 1999, my first research effort was to investigate the growth mechanism of semiconductor nanowires. Initially, we tried to explore in situ growth of Ge nanowires; we observed the process of VLS growth using an in situ high temperature transmission electron microscope (TEM) ${ }^{5}$ in this case we were investigating the nucleation of a Ge nanowire, using a gold nanoparticle heated to $800^{\circ} \mathrm{C}$ as the catalyst. Based on the Au-Ge phase diagram, at $800^{\circ} \mathrm{C}$, we can first observe an alloying process that leads to the liquid formation; with more Ge vapor incorporation, the system eventually moves into a biphasic region, and we can observe the nucleation event in real time. The creation of this solid-liquid interface is the starting point for one-dimensional crystal growth; this underpins the fundamental nucleation step at the nanometer scale for all of the VLS processes.

In the 1990s, there were very few papers published in the semiconductor nanowire field, but there has been a rapid increase in the past 10 years, and nowadays there are thousands of papers published every year on this subject, with hundreds of research groups now active in this exciting area. achieve these functionalities either in air or in a liquid. ${ }^{9}$ This is quite powerful, as we have basically developed a nanoscopic light source that can be potentially used in a liquid medium.

Recently, we have been trying to utilize these nanoscopic light sources for endoscopy at the single living cell level. ${ }^{10}$ We attached a single nanowire onto an optical fiber so that we could deliver light directly to the nanowire waveguide and then use the waveguided end emission to perform imaging at the single-cell level. These nanowires are very robust, do not fracture easily, and can be bent, so they are stable enough to be used in single-cell endoscopy. As illustrated in Figure 2, the nanowire had quantum dots attached to the surface that were cleaved by UV light. After cleaving, the quantum dots were delivered to a single cell. As an example, we can deliver the quantum dots into the cytoplasm and directly to the nucleus. It is

\section{Nanophotonics}

In the last decade, we have seen many important discoveries in a couple of different directions, such as nanoelectronics and photonics, and next I will describe some of the work we did in these areas. In 2000, we discovered that a single-crystalline semiconductor nanowire can serve as a nanoscopic laser cavity (Figure 1). ${ }^{6}$ This is basically a Fabry-Pérot cavity, and Figure $1 \mathrm{~b}$ shows the far-field emission patterns from single nanowires, the power-dependent laser spectra from these individual nanowires, and the integrated intensity as a function of the incident power. For the last couple of years, we have extended our work on photonics from nanolasers to subwavelength waveguides, as well as using a single nanowire to perform nonlinear optical mixing. ${ }^{7,8}$ Due to the high refractive index of these nanostructures, we can
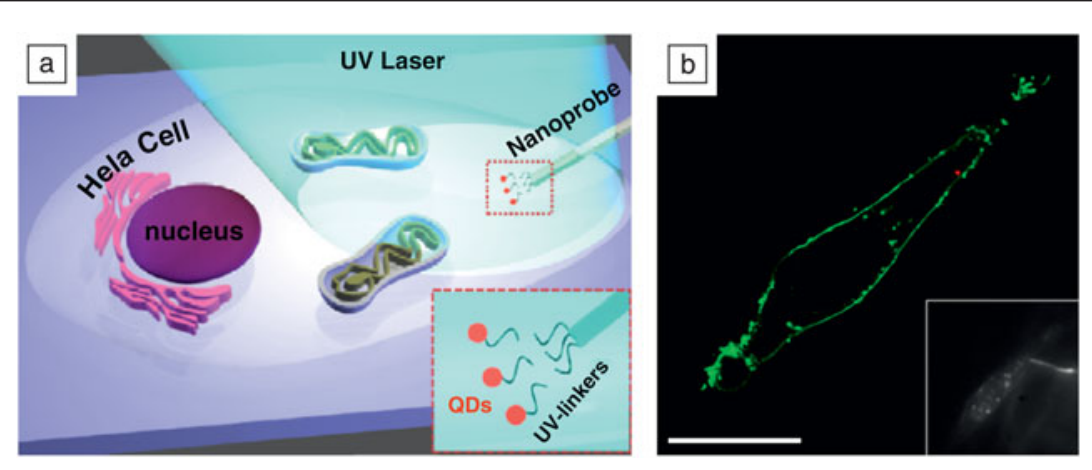

Figure 2. Nanowire single-cell endoscopy. (a) Schematic of the spatiotemporal delivery of quantum dots (QDs) into a living cell using a photoactivated nanowire endoscope. Inset shows QDs were conjugated to the nanowire via photocleavable linkers. (b) Fluorescence confocal image of a Hela cell after QD delivery showing nanoprobe delivered QDs (red dot) sitting in the cytoplasm within the cell membrane (green), which was labeled with Alexa Fluor 488 conjugate of WGA. A $488 \mathrm{~nm}$ laser was used to excite both the cell membrane stain and the QDs. The inset is a dark-field image of the cell during QD delivery with a nanowire endoscope (bright line). Scale bar is $20 \mu \mathrm{m}$. Reprinted with permission from Reference 9. ()2012, Nature Publishing Group. 
then possible to perform high spatial-resolution imaging. Using the nanowire as a scanning probe in a liquid environment is a powerful imaging tool. I believe that in the next few years, we will continue to see many new developments that integrate all of these active nanoscopic optical components into integrated nanophotonic circuits and also use the nanowire probe at the single-cell level for targeted delivery, imaging, or even stimulating single living cells.

\section{Thermoelectric energy conversion}

I want to focus now on how these nanoscale one-dimensional structures can be used for terawatt energy conversion and storage. We all know that the amount of energy required worldwide is on the scale of terawatts, and the percentage of renewable energy in the current energy portfolio is very limited. Many research groups are now working on ways to save energy or convert renewable energy. My group approaches this problem from two different directions. The first relates to saving energy by developing nanostructured silicon thermoelectrics for waste heat recovery, and the second, more recent effort, involves developing nanostructures for solar energy conversion, either directly into electricity or into liquid fuels through artificial photosynthesis.

If we examine the performance of power plants delivering $15 \mathrm{TW}$ of power through fossil fuels, we find the total energy expended is $38.6 \mathrm{TW}$ with $40 \%$ efficiency for the heat engine. This means that about $20 \mathrm{TW}$ is lost as waste heat to the environment. The question is whether we can develop a technology to recover some of this waste heat energy? A thermoelectric material provides a suitable strategy for this. If we had a thermoelectric waste heat recovery module with an efficiency of $3 \%$, we could recover up to $1 \mathrm{TW}$ of energy; this is a potential solution to the terawatt energy challenge, provided we can find the right thermoelectric material to achieve this.

We started to work in this field about 10 years ago, together with Arun Majumdar in the Mechanical Engineering Department at Berkeley. We first examined the thermal transport in a single-crystalline silicon nanowire. For any thermoelectric material, the important figure of merit is the dimensionless parameter ZT, which is related to the Seebeck coefficient, the electrical conductivity, and the thermal conductivity of the material. A high ZT value means high energy conversion efficiency. We considered using one-dimensional structures to control thermal transport, essentially to suppress the thermal conduction, while allowing all the carriers, electrons, or holes to transport and maintain a reasonable level of the Seebeck coefficient. The design principle, for $\mathrm{Si}$ in particular, was to choose a wire diameter that is smaller than the phonon mean-free path (200-300 nm) but larger than the electron meanfree path (1-2 nm). We measured the thermal conductivity of individual nanowires using a suspended microelectromechanical system (MEMS) device by heating one end and then measuring the thermal transport to the other end of the MEMS platform. By measuring the steady-state temperature on the other end, we can calculate the thermal conductance of the nanowire thermal bridge, normalize it to the nanowire cross-section, calculate the thermal conductivity, and then examine the size dependence of these semiconductor structures.

The initial results were very promising. ${ }^{11}$ This was the first study of size-dependent thermal conductivity of Si nanowires. The thermal conductivity of the Si nanowires was suppressed by an order of magnitude compared to bulk Si. By simply decreasing the diameter of the nanowire, we could decrease the thermal conductivity through phonon-boundary scattering. However, the thermal conductivity was still quite high, typically at the level of $10 \mathrm{Wm}^{-1} \mathrm{~K}^{-1}$, which is not sufficient for a good thermoelectric material. Later, after several years of exploring various strategies to further decrease the thermal conductivity, we started investigating a solution-based process for the production of silicon nanowires with a rough surface, which was found to be critical for further thermal conductivity reduction. ${ }^{12}$ This is a simple etching process that creates a rough surface on the silicon nanostructure. It led to the large scale production of rough silicon nanowires; they can now be made on six-inch wafers. The nanoscopic surface roughness turns out to be the key structural feature to further decrease the thermal conductivity, without significantly decreasing the Seebeck coefficient and electrical conductivity of these nanowires. These nanowires now have values of ZT that are two orders of magnitude greater than that of bulk $\mathrm{Si}$ and reach a value of 0.6 , as shown in Figure 3.

In 2009, together with a Berkeley graduate student, I founded the Alphabet Energy Company, and we are already producing modules using these Si nanostructures. The company currently employs 16 people and is continuing to hire, so nanowire technology is indeed creating jobs. We will have our 
first pilot thermoelectric testing in mid-2012. The prospect of using these nanostructured silicon thermoelectrics for waste heat recovery is real.

\section{Conversion and storage of renewable energy}

I will now concentrate on the semiconductor nanostructures required to convert and store renewable solar energy. Since we are aiming to operate at the terawatt level, the amount of solar energy required is enormous, and this must be achieved with reasonable efficiency and at a reasonable cost. Before discussing the use of nanowires for this purpose, I want to emphasize some of the fundamental materials requirements for designing solar energy conversion devices. First is the use of the earth's abundant elements, which will be absolutely necessary if we are to work on the terawatt scale; and second, whether we are developing solar-cell devices or an artificial photosynthetic system, we need to use less-energy-intensive processes to make these materials and to create the necessary systems. Where possible, we want to use mild chemistry or mild processing conditions and minimize the energy used to create these energy conversion devices; this applies to both photovoltaic (PV) applications and artificial photosynthesis.

We started our research on nanowire-based solar cells about seven years ago. The first example was to use a dye-sensitized cell (DSC) as a test platform, with single-crystalline nanowires as the electrodes, replacing the traditional $\mathrm{TiO}_{2}$ nanoparticle percolation network. ${ }^{13}$ One of the major advantages of using the single-crystalline nanowire conduction channel in a DSC cell is that electron diffusivity is enhanced, compared to the nanoparticle percolation networks. In recent years, there have been various research efforts utilizing nanowires in composite solar cells or in a core-shell gemotry. Nanowire structures have many benefits; it is fairly straightforward to design a suitable interface to facilitate charge separation, and the charge collection can also be quite efficient. In addition, the core-shell nanowire geometry has the advantage of orthogonalized light absorption and charge separation, a feature quite important for material systems with short minority carrier diffusion length. For nanowire arrays with a suitable periodicity, we can introduce a light-trapping mechanism so that less material will be needed for light collection.

With all the benefits of using nanowires as the PV active elements, we should not forget that we are also introducing an important potential limitation - the large junction area. These large interfacial areas in nanostructured solar cells naturally introduce significant trapped states and various channels for carrier recombination. If we examine the performance of these nanostructured PVs, we typically find that the fill factor is low, and the open circuit voltage $\left(V_{\text {oc }}\right)$ is low. This is intrinsically related to the high junction surface area solar cell and is particularly obvious in the nanowire polymer composite solar cell, where low fill factors and $V_{\text {oc }}$ were typically reported. To mitigate this problem, we developed a simple solution procedure to produce high quality nanowire core-shell solar cells with excellent fill factor and $V_{\text {oc }}{ }^{14}$ We first make CdS nanowires in solution, then we use a mild cation-exchange chemistry to produce the core-shell nanostructure, as shown in Figure 4. This cation exchange chemistry was developed in Paul Alivisatos's group at the University of California, Berkeley. All that is required is to dip a nanowire for a couple of seconds in a copper solution at $50^{\circ} \mathrm{C}$; the core-shell nanowires can then be fabricated into an asymmetrical junction to measure PV performance.

It is interesting to note that we can create the interface using mild processing conditions. The resulting interface is highly coherent, with a minimum amount of defects or dislocations. The $\mathrm{CdS}-\mathrm{Cu}_{2} \mathrm{~S}$ interface is illustrated in Figure $4 \mathrm{~b}-\mathrm{c}$. The heterojunction prepared by this method is atomically well-defined with few interface defects, which enables excellent charge separation and minimal minority carrier recombination. As a result, our nanowire PV device shows excellent $V_{\text {oc }}$ and fill factors. Figure $4 \mathrm{e}$ shows the I-V curve from the individual nanowires, and the current output is limited by absorption in the visible region, which is limited in turn by the thickness of the $\mathrm{Cu}_{2} \mathrm{~S}$ shell (Figure 4f). However, the most important message here is that the fill factor and $V_{\mathrm{oc}}$ of this device are even better than those for traditional thin films. The insight we gained here is that we can indeed create highly coherent interfaces using mild chemistry and less-energy-intensive processes. Such a coherent interface is the key structural feature that could lead to high fill factor and $V_{\text {oc }}$ for nanostructured solar cells. I believe that with this strategy, we will be able to push the efficiency above $10 \%$ by adding more visible light absorbing $\mathrm{Cu}_{2} \mathrm{~S}$.

\section{Artificial photosynthesis}

For PVs, we will have to worry about the energy storage issue because of the intermittency of sunlight irradiation. It would be much more appealing if somehow we can convert the solar energy directly into liquid fuels. This is artificial photosynthesis, where we try to mimic what is happening in nature: to convert solar energy and store it in chemical form, possibly in the form of hydrogen, methanol, or even gasoline. In our daily life, we use gasoline in cars, and as a result, $\mathrm{CO}_{2}$ is emitted into the environment. Artificial photosynthesis attempts to convert the $\mathrm{CO}_{2}$ and water back into liquid fuel, which will be a truly carbon neutral process. Once again, in this artificial photosynthesis process, we have to emphasize the need to use earth abundant elements and more energy efficient processes.

If we examine the chemical reactions required to generate solar fuels, either by water splitting or $\mathrm{CO}_{2}$ reduction (where there are many possible products), they are all "uphill" reactions, not spontaneous; we will need solar energy as the energy input and catalysts to drive these reactions. Therefore, in order to generate solar fuel by artificial photosynthesis, we need two things: the first is quite similar to PVs; we need to have the semiconductor and the junctions for light harvesting and charge separation. Then we need to utilize the charge carriers to run the reactions, instead of extracting them as electricity as in solar 


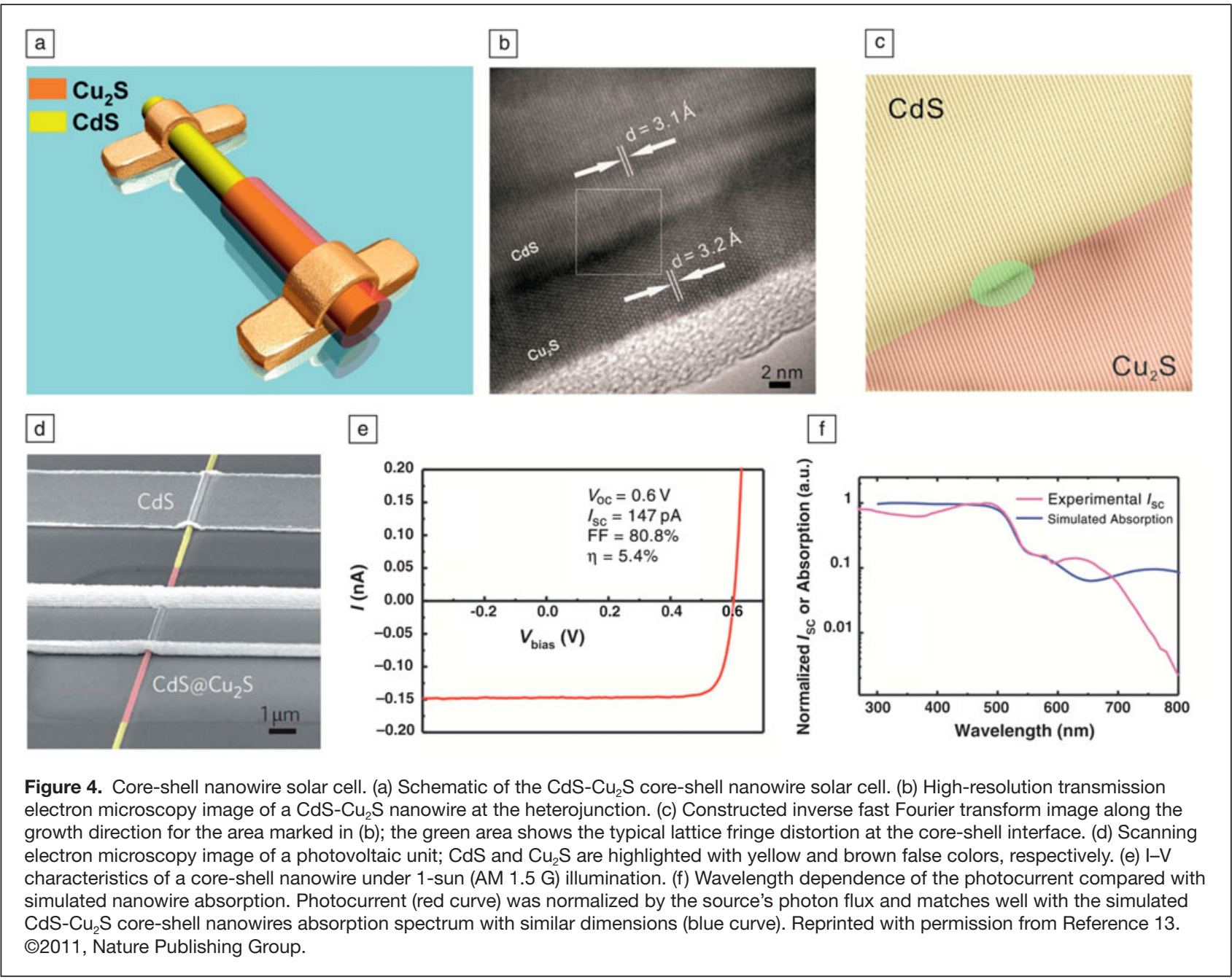

cells. If we consider the materials challenges in developing this artificial photosynthesis system, we find many of them are relevant to the overall work performed by the materials research community at large. Two examples are photocathode materials for hydrogen and $\mathrm{CO}_{2}$ reduction and photoanode materials for water oxidation. The photoanode materials are particularly challenging, and we do not have a solution at this moment. We also need to develop better catalysts for water reduction, $\mathrm{CO}_{2}$ reduction, and water oxidation. In order to balance the ion flux during this artificial photosynthetic process, we also need an ion conductive membrane that is gas impermeable, mechanically stable, and optically transparent, and this must sit between the light absorbing photoanode and photocathode.

Semiconductor nanowires, with their high charge mobility and high surface area, are good candidates to serve as the scaffold for the entire artificial photosynthetic system. Solar water-splitting research commenced with Fujishima and Honda using $\mathrm{TiO}_{2}{ }^{15}$ which is a UV absorbing material. Although the basic concept works quite well, this will never result in a high efficiency photoelectrochemical (PEC) cell because the fraction of UV photons in the solar spectrum is limited. Nozik ${ }^{16}$ proposed the PEC diode concept based on the concept of a coupled photocathode and anode, with an ohmic contact in between so that minority carriers can be used on both sides to run the water reduction and the water oxidation for complete solar water splitting. This two-photon process essentially mimics the photosynthetic process in nature; now we can place two small bandgap materials back-to-back in a diode configuration and make use of the UV and visible spectrum. The major advantage here is the increased photocurrent from the coupled photocathode and anode system, compared to the single bandgap structure. The single bandgap structure is limited to the UV region, while in the two-bandgap system we can make use of the entire solar spectrum, and current density at a $20 \mathrm{~mA} / \mathrm{cm}^{2}$ level is possible. Our main goal was to capture the entire solar spectrum and use it for solar fuel conversion.

We have a couple of good candidates for the photocathodes. The Si nanowire array is certainly very promising; Figure 5 shows black-colored silicon nanowire arrays in the form of $2 \mathrm{~cm} \times 2 \mathrm{~cm}$ wafers. We can make these ordered nanowire arrays shown in Figure 5b, and decorate them with water reduction catalysts such as $\mathrm{Pt}$ or $\mathrm{MoS}_{2}$ nanoclusters. These devices have reasonable photocurrent output up to $20 \mathrm{mAcm}^{-2}$, and we can implement the "half reaction" of hydrogen generation 

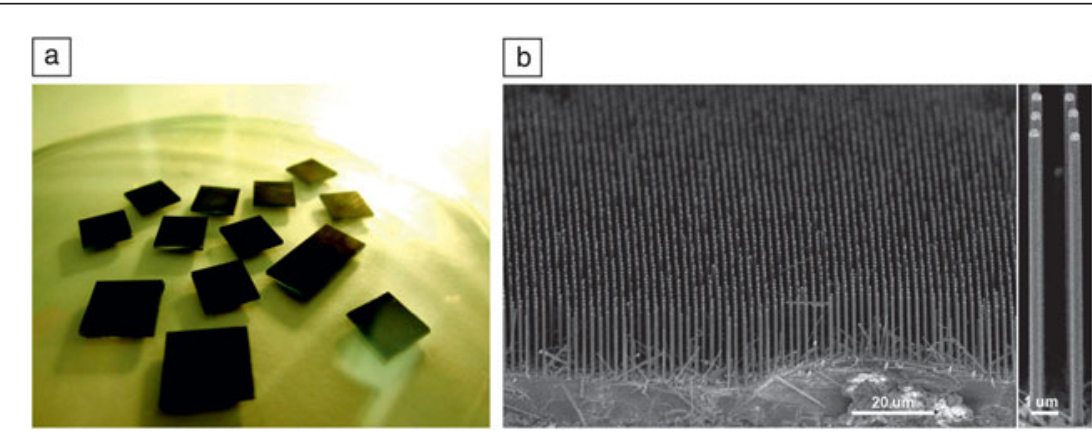

Figure 5. Silicon nanowire array photocathode. (a) Digital photograph of the silicon nanowire array on $2 \mathrm{~cm} \times 2 \mathrm{~cm}$ wafers. (b) Cross-sectional scanning electron microscopy images of the silicon nanowire arrays.

this way. ${ }^{22}$ More recently, we have also investigated possible photocathodes for $\mathrm{CO}_{2}$ reduction. For the $\mathrm{CO}_{2}$ reduction, we chose $\mathrm{GaP}$ because $\mathrm{CO}_{2}$ redox potential is more negative than the hydrogen redox potential. For this purpose, we developed a surfactant-free solution process to generate a large quantity of single-crystalline GaP nanowires (Figure 6). ${ }^{17}$ We can now produce these $\mathrm{GaP}$ nanowires without an organic coating, and we can control the doping by adding a zinc precursor as well. We believe that a combination of $\mathrm{Mo}_{3} \mathrm{~S}_{4}$ cubane molecular catalysts and high surface area GaP nanowires can lead to a very promising photocathode.

The anode, however, presents more of a problem, because we have a very limited choice of meterial. The best candidate is $\mathrm{Si}$-doped $\mathrm{Fe}_{2} \mathrm{O}_{3}$ from Michael Grätzel's lab at the Ecole Polytechnique de Lausanne in Switzerland, for which photocurrent is typically in the single digit $\mathrm{mA} / \mathrm{cm}^{2}$ range. ${ }^{18}$ Our group has been working on $\operatorname{In}_{x} \mathrm{Ga}_{1-\mathrm{x}} \mathrm{N}$ nanostructures with a tunable bandgap from $3.3 \mathrm{eV}$ to $1 \mathrm{eV}{ }^{19}$ We are hopeful that, by using a tunable bandgap material to cover the solar spectrum, we will be able to develop a useful photoanode. The initial testing on the photocurrent output is quite disappointing, in the range of $50 \mu \mathrm{A} / \mathrm{cm}^{2}$, far below that from what we would expect based on their visible bandgap. ${ }^{21}$ We have short carrier lifetime issues and extraction issues here, and clearly these $\mathrm{In}_{\mathrm{x}} \mathrm{Ga}_{1-\mathrm{x}} \mathrm{N}$ nanostructures need to be optimized for photoanode applications.

Finally, I will briefly discuss the way we plan to implement this two-bandgap configuration. We have two schemes: the first is an asymmetric $\mathrm{Si}-\mathrm{TiO}_{2}$ core-shell model system; the second is based on bilayer nanowire fabrics. In the first case, the $\mathrm{Si} / \mathrm{TiO}_{2}$ asymmetrical junction represents a fully integrated cathode and anode in a single nanowire (Figure 7): $;{ }^{20} \mathrm{Si}$ can be decorated once again by the reduction catalyst, and these arrays can be used directly for water splitting. This process is based on CVD; large reactors can be used to make larger arrays. It is, however, quite energy intensive to create these asymmetrical junctions. We are also investigating silicon nanowires decorated by high surface area $\mathrm{InGaN}$ nanorods (Figure 8), ${ }^{21}$ and this will be our model system for balancing the photocurrent from the anode and cathode. However, contact between these two materials remains to be optimized.

We recently embarked on the concept of bilayer nanowire fabrics for PEC applications; everything here is processed in solution, and the fundamental idea is quite similar to the asymmetric junction. Basically, we want to directly couple the photoanode and photocathode, both made of nanowire mesh; one side will perform the water oxidation, the other side will be used for water reduction, and a proton transport medium will

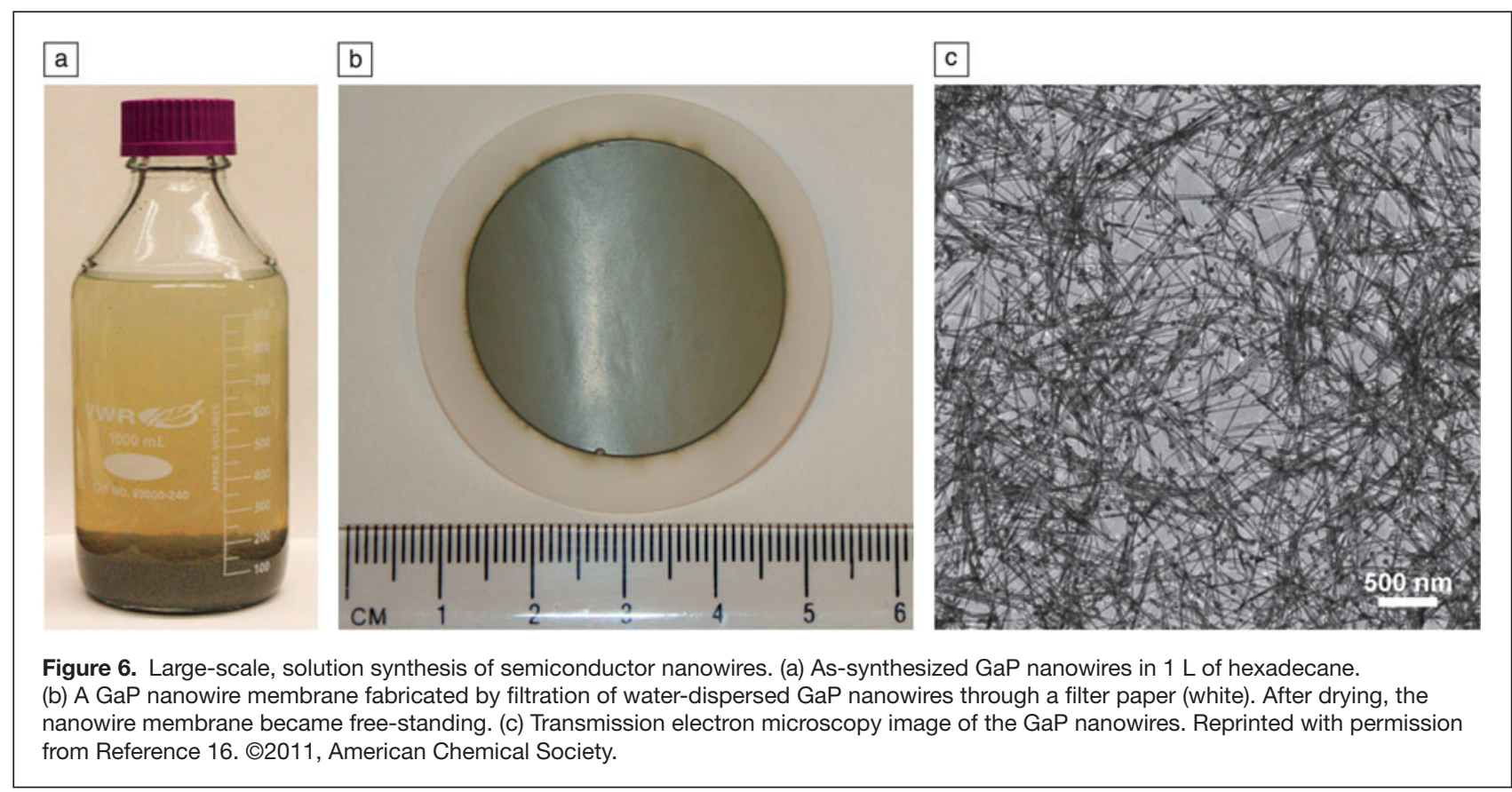



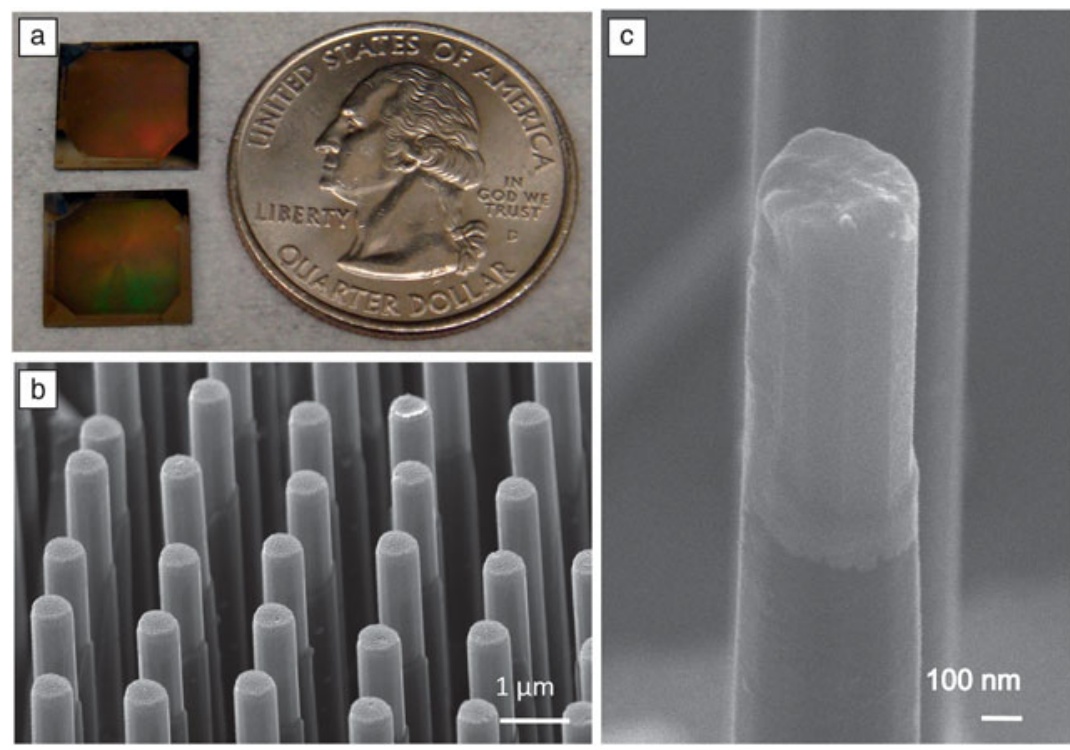

Figure 7. $\mathrm{Si} / \mathrm{TiO}_{2}$ asymmetric core-shell nanowire array. (a) Optical image of asymmetric nanowire array made using soft lithography, showing incandescent color due to array periodicity. Scanning electron microscopy images of an asymmetric nanowire array (b) and an individual nanowire (c). Reprinted with permission from Reference 19. (O2011, American Chemical Society.
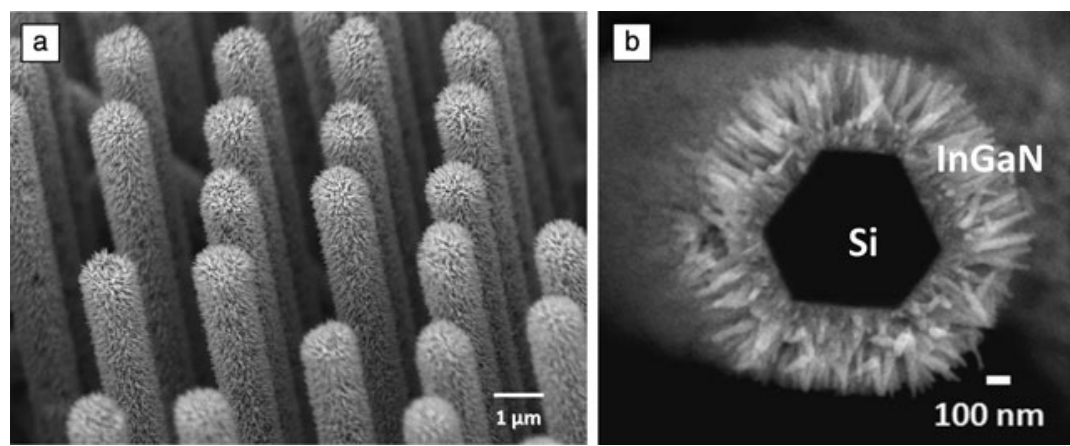

Figure 8. High surface area Si/InGaN nanowire photoanodes. Scanning electron microscopy images of hierarchical $\mathrm{Si} / \mathrm{In}_{x} \mathrm{Ga}_{1-x} \mathrm{~N}$ nanowire arrays on a $\mathrm{Si}$ (111) substrate with $X=0.08 \sim 0.1$ (a). A fractured wire reveals the cross-section (b) showing that InGaN nanowires grow vertically from the six $\mathrm{Si}$ wire facets. Reprinted with permission from Reference 20. (02012, American Chemical Society.

be placed in between. We can now make two-inch diameter discs by laminating two layers of nanowire materials together, for example, a $\mathrm{WO}_{3}$ or $\mathrm{TiO}_{2}$ nanowire mesh on top and a $\mathrm{GaP}$ nanowire mesh underneath, where one is the photocathode and one is the photoanode. Of course the bandgap and photocurrent output do not match at this point. We are trying to use this as a model system to demonstrate whether such a bilayer nanowire fabric idea can work. The initial results are promising. The main problem right now is the urgent need to discover better anode materials to replace $\mathrm{TiO}_{2}$ or $\mathrm{WO}_{3}$. Above all, we believe that these high surface area semiconductor nanowires, with their high carrier mobilities, will eventually be part of the artificial photosynthesis system as these nanostructures enable the stacking of the catalysts in the third dimension, and effectively relax the stringent requirements for catalysts with high turnover frequency.

\section{Summary}

Over the past 10 years, we have continued to develop the science and technology of semiconductor nanowires. As of today, nanowires with different sizes, growth directions, compositions, and heterojunctions can be rationally designed, synthesized, and assembled. We have already seen major progress in many different areas of nanowire research (e.g., electronics, photonics); we can be confident that we will continue to see many more fundamental new discoveries and science based on this unique class of nanoscale building blocks. Considering their unique structural, optical, and electrical properties, we expect that these nanostructures will have a significant impact on large scale clean energy conversion and storage technologies. In the meantime, the future of nanowire technology will be largely dependent on how well we can balance the issues of cost, performance, and stability of nanowire-based devices and systems.

\section{Acknowledgments}

I wish to thank my research group for their hard work. I would also like to acknowledge the generous continued support from the Department of Energy, Office of Basic Energy Sciences over the last 10 years, without which much of the progress I discussed would be impossible.

\section{References}

1. R.S. Wagner, W.C. Ellis, Appl. Phys. Lett. 4, 89 (1964). 2. Y.N. Xia, P.D. Yang, Y.G. Sun, Adv. Mater. 15, 353 (2003).

3. P. Yang, C.M. Lieber, Science 273, 1836 (1996)

4. A.M. Morales, C.M. Lieber, Science 279, 208 (1998).

5. Y. Wu, P. Yang, J. Am. Chem. Soc. 123, 3165 (2001)

6. M. Huang, S. Mao, H. Feick, H. Yan, Y. Wu, H. Kind, E. Weber, R. Russo, P. Yang, Science 292, 1897 (2001).

7. M. Law, D. Sirbuly, J. Johnson, J. Goldberger, R. Saykally, P. Yang, Science 305, 1269 (2004).

8. Y. Nakayama, P.J. Pauzauskie, A. Radenovic, R.M. Onorato, R.J. Saykally, J. Liphardt, P. Yang, Nature 447, 1908 (2007).

9. D.J. Sirbuly, M. Law, P. Pauzauskie, H. Yan, A.V. Maslov, K. Knudsen, R.J. Saykally, P. Yang, PNAS 102, 7800 (2005).

10. R. Yan, J. Park, Y. Choi, C. Heo, S. Yang, L.P. Lee, P. Yang, Nat. Nanotechnol. 7, 191 (2012).

11. D. Li, Y. Wu, P. Kim, L. Shi, N. Mingo, Y. Liu, P. Yang, A. Majumdar, Appl. Phys. Lett. 83, 2934 (2003).

12. A.I. Hochbaum, R. Chen, R.D. Delgado, W. Liang, E.C. Garnett, M. Najarian, A. Majumdar, P. Yang, Nature 451, 163 (2008).

13. M. Law, L. Greene, J.C. Johnson, R.J. Saykally, P. Yang, Nat. Mater. 4, 455 (2005).

14. J. Tang, Z. Huo, S. Brittman, H. Gao, P. Yang, Nat. Nanotechnol. 6, 568 (2011).

15. A. Fujishima, K. Honda, Nature 238, 37 (1972)

16. A.J. Nozik, Appl. Phys. Lett. 30, 567 (1977).

17. J. Sun, C. Liu, P. Yang, J. Am. Chem. Soc. 133, 19306 (2011). 
18. A. Kay, I. Cesar, M. Grätzel, J. Am. Chem. Soc. 128, 15717 (2006). 19. T. Kuykendall, P. Ulrich, S. Aloni, P. Yang, Nat. Mater. 6, 951 (2007). 20. C. Liu, Y. Hwang, H.E. Jeong, P. Yang, Nano Lett. 11, 3755 (2011). 21. Y. Hwang, C. Wu, C. Hahn, H. Jeong, P. Yang, Nano. Lett. 12, 1678 (2012). 22. E. Garnett, P. Yang, Nano Lett. 10, 1082 (2010).

23. D.J. Sirbuly, M. Law, H. Yan, P. Yang, J. Phys. Chem. B 109, 15190 (2005).

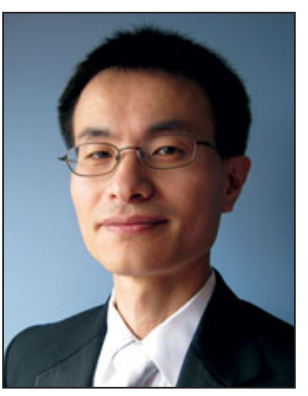

Peidong Yang is a professor in the Department of Chemistry, Materials Science and Engineering, the S.K. and Angela Chan Distinguished Chair in Energy, and a senior faculty scientist at the Lawrence Berkeley National Laboratory (LBNL). He received a BS degree in chemistry from the University of Science and Technology of China (1993) and a PhD degree in chemistry from Harvard University (1997). His postdoctoral research was at the University of California, Santa Barbara, after which he joined the Department of Chemistry faculty at the University of California, Berkeley (1999). He founded the nanoscience subdivision within the American Chemical Society (ACS) and also co-founded two startups, Nanosys Inc. and Alphabet Energy Inc. In addition, he is an associate editor for the Journal of the American Chemical Society and serves on the editorial advisory boards for a number of journals, including Accounts of Chemical Research and Nano Letters. His main research interest is in the area of one-dimensional semiconductor nanostructures and their applications in nanophotonics and energy conversion. Yang is the recipient of numerous awards and recognitions. He can be reached at the Department of Chemistry, University of California, Berkeley, Materials Science Division, Lawrence Berkeley National Laboratory, Berkeley, CA 94720, USA; email p_yang@uclink.berkeley.edu.

\section{Influence the Future of Your Society}

\section{COMING SOON_Election of MRS}

\section{Officers \& Board Members}

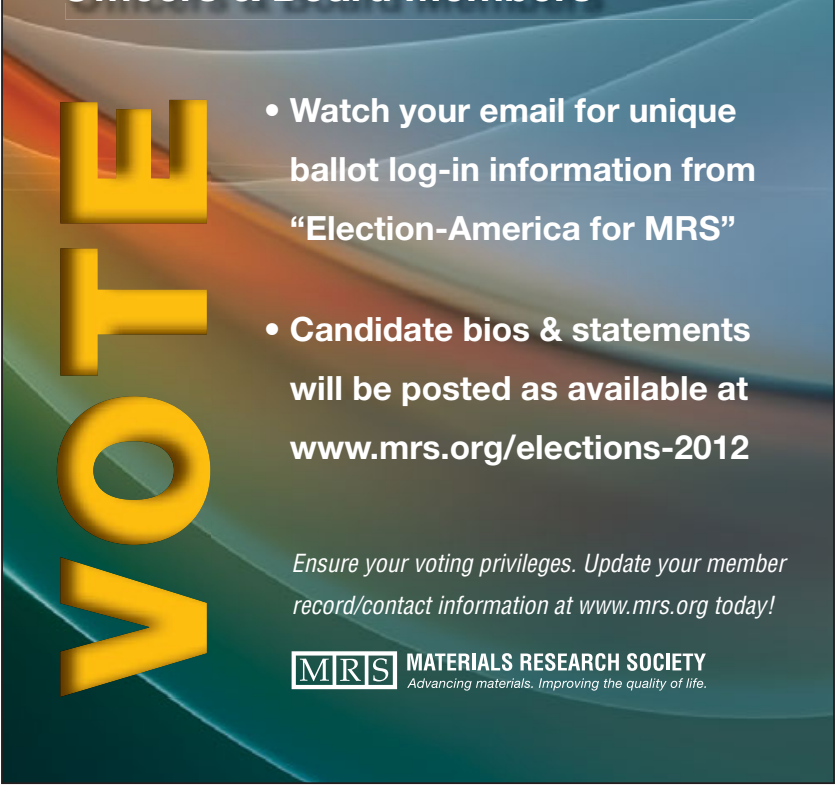

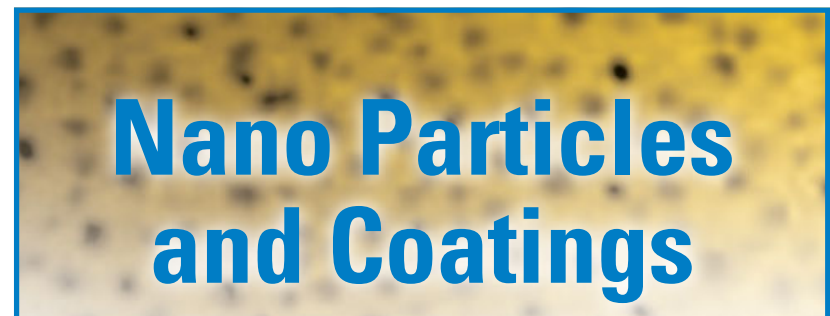

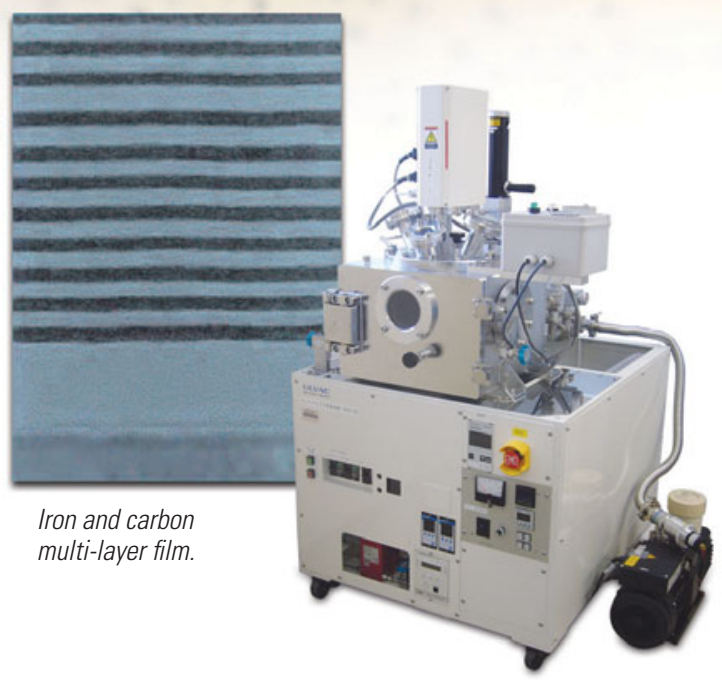

The ULVAC Arc Plasma Deposition System (APD) produces extremely smooth thin films and uniformly sized nano particles. The APD System deposits magnetic, DLC and metal films in R\&D, material science, fuel cell and automotive applications.

The APD System delivers:

- Extremely smooth ultra-thin films -0.01 to $0.3 \mathrm{~nm} / \mathrm{sec}$

- Size-controlled nano particles - $1 \mathrm{~nm}$ to $30 \mathrm{~nm}$ dia.

- Dense film formation without process gas

- Small target size: $10 \mathrm{~mm}$ dia. $\times 17 \mathrm{~mm}$

- Uniformity $+/-10 \%$ over $50 \mathrm{~mm}$ diameter coated area

\section{Need Nano particles or coatings? Call 800-99ULVAC or email sales@us.ulvac.com.}

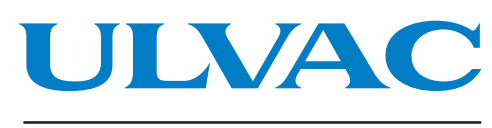

Methuen, MA • Tel: 978-686-7550 sales@us.ulvac.com・www.ulvac.com 